PROCEEDINGS OF THE

AMERICAN MATHEMATICAL SOCIETY

Volume 128, Number 8, Pages 2203-2212

S 0002-9939(00)05593-3

Article electronically published on February 25, 2000

\title{
ON EULER PRODUCTS ASSOCIATED WITH NONCUSPIDAL METAPLECTIC FORMS
}

\author{
SHAMITA DUTTA GUPTA AND XIAOTIE SHE
}

(Communicated by Dennis A. Hejhal)

\begin{abstract}
In this paper, we obtain an Euler product with functional equation associated to a noncuspidal metaplectic form $f$ on the double cover of $G L(2)$. Zagier's idea of Rankin-Selberg method is used to define the convolution of $f$ and the $\theta$-function.
\end{abstract}

\section{INTRODUCTION}

If $f$ is a holomorphic modular form of half-integral weight $k / 2$, Shimura [16] showed that there is an automorphic form of integral weight $2 k-2$ associated to $f$. This phenomenon is usually referred to as the Shimura Correspondence. Here is the outline of Shimura's original proof. Suppose $f(z)=\sum a(n) q^{n}$ is the Fourier expansion of $f$, which is an eigenform of the Hecke operators $T_{p^{2}}$. Then the Rankin Selberg integral

$$
\int f(z) \overline{\theta(z)} E(z, s) d z
$$

has the functional equation and the Euler product of the form

$$
\prod_{p}\left(1-\lambda_{p} p^{-s}+p^{k-2-2 s}\right)^{-1}
$$

where $\theta(z)$ is the classical theta function and $E(z, s)$ is an Eisenstein series, $\lambda_{p}$ is the eigenvalue of $f$ under $T_{p^{2}}$. By means of the converse theorem, Shimura showed that the above Euler product is the L-function of automorphic form of integral weight $2 k-2$.

There are few other approaches towards the Shimura Correspondence, such as, as a case of the theta correspondence [17], 14, [7, use of Selberg's trace formulas [5], 6].

On the other hand, Shimura's original method also has been used to obtain Euler products with functional equations in other metaplectic settings. The analytic property of these Euler products, combined with the generalized converse theorems, suggests the generalized Shimura correspondence. The work of Gelbart and Piatetski-Shapiro [9] deals with the double cover of all forms over all base fields. Bump and Hoffstein worked on metaplectic forms on the cubic cover of $G L(3)$ [1]

Received by the editors September 8, 1998.

1991 Mathematics Subject Classification. Primary 11F55; Secondary 11F30.

Key words and phrases. Eisenstein series, automorphic forms, Fourier expansions, Euler products, functional equations. 
and on the $n$-cover of GL(2) 2]. Similar results are also obtained by Friedberg and Wong [8] over the 2-cover of $G S p(4)$ and by Goetze [10] over the 3-cover of $G S p(4)$.

All above mentioned Euler products with functional equations handle only cusp forms. In this paper, we will extend the construction in a new direction. We will obtain Euler products with functional equation associated to noncusp forms. To illustrate our idea, we will consider a noncuspidal metaplectic form on the double cover of $G L(2, \mathbb{C})$, but the method may be generalized to deal with other more complicated settings. The Euler product exists in this noncuspidal case, due to the same reason as the cuspidal case. The Hecke operators force the Fourier coefficients to satisfy a certain relation; this relation leads to the convolution having an Euler product. But as the form under consideration is noncuspidal, the convolution as in (0.1) is not well defined; we will adjust the convolution using Zagier's idea for the Rankin-Selberg method for functions not of rapid decay 18. Zagier's method for functions not of rapid decay has been widely used to handle convolutions which involve metaplectic Eisenstein series and theta series, as they are not cuspidal and their Fourier coefficient contains number theoretic information [11, 15. Clear formulations and rigorous proofs of the method in contexts other than Zagier's may be found in [13, [3, 4].

It should be pointed out that, in this situation, the $p$-factor of the Euler product, unlike the cuspidal case, may split. For example, when $f=\theta$ itself, we have

$$
\lambda_{p}=(\mathbb{N} p)^{\frac{1}{2}}+(\mathbb{N} p)^{-\frac{1}{2}},
$$

and

$$
1-\lambda_{p} x+x^{2}=\left(1-(\mathbb{N} p)^{\frac{1}{2}} x\right)\left(1-(\mathbb{N} p)^{-\frac{1}{2}} x\right) .
$$

Thus, in this case, the Euler product does not imply the Shimura correspondence. Further we point out that, in cases like $f=\theta$, it is possible to compute $\lambda_{p}$ explicitly. Thus our result may be obtained by explicit computation.

The method outlined in this work, when applied to other more complicated settings, such as $n$-fold covers of $G L(r)$, might help us to understand the $\theta$-series and Hecke operators on those settings better. For example, we suspect that working on the 4-fold cover of $G L(4)$ and the 4-fold cover of $G L(2)$ might reveal some information about the Fourier coefficients of the $\theta$-series of the 4-cover of $G L(2)$, which at this point is not yet completely determined.

As most of the ideas and computations used here are well documented, we will omit most of the details of the proofs. But the statements of our results are precise.

\section{Notation}

Let $K=Q(i)$ and $\mathcal{O}=Z[i]$ be the ring of integers of $K$. Let $\lambda=1+i$. The ring $\mathcal{O}$ is the principal ideal domain and every integral ideal of $\mathcal{O}$ which is prime to $\lambda$ has an unique generator which is congruent to 1 modulo $\lambda^{3}$. This generator is called primitive. For any element of $K$, or an ideal of $\mathcal{O}$, we will denote its absolute norm by $\mathcal{N}$, and the cardinality of the multiplicative group of $\mathcal{O} / a \mathcal{O}$ by $\phi(a)$.

If $a, b$ are coprime elements of $\mathcal{O}$, and $\lambda \backslash \backslash$, let $\left(\frac{a}{b}\right)$ denote the quadratic symbol. For a complex number $z$, let $e(z)=e^{2 \pi i \operatorname{Re}(z)}$. For a primitive integer $d$ and $n \in \mathcal{O}$, we define the Gauss sum as

$$
g(n, d)=\sum_{c(\bmod d)}\left(\frac{c}{d}\right) e\left(\frac{n c}{d}\right) .
$$


Here is the summary of properties of this Gauss sum:

Proposition 1.1. a) Suppose that $m, d$ are coprime; then $g(m n, d)=\left(\frac{m}{d}\right) g(n, d)$.

b) Suppose that $d_{1}, d_{2}$ are coprime; then $g\left(n, d_{1} d_{2}\right)=g\left(n, d_{1}\right) g\left(n, d_{2}\right)$.

c) Suppose that $l$ is even and $p$ is prime; then

$$
g\left(p^{k}, p^{l}\right)= \begin{cases}\phi\left(p^{l}\right) & \text { if } k \geq 1 \\ -\mathbb{N} p^{k} & \text { if } k=l-1 \\ 0 & \text { otherwise. }\end{cases}
$$

d) Suppose that $l$ is odd and $p$ is prime; then

$$
g\left(p^{k}, p^{l}\right)= \begin{cases}\mathbb{N} p^{k+1 / 2} & \text { if } k=l-1 \\ 0 & \text { otherwise. }\end{cases}
$$

Let $\mathcal{H}=G L(2, \mathbb{C}) / \mathbb{C}^{\star} U(z)$ be the hyperbolic 3 -space and suppose $G L(2, \mathbb{C})$ acts on $\mathcal{H}$ by left multiplication. We have the following coset representatives for $\mathcal{H}$, given by Iwasawa decomposition:

$$
\left\{z=y^{-\frac{1}{2}}\left(\begin{array}{ll}
y & x \\
0 & 1
\end{array}\right) \mid x \in \mathbb{C}, 0<y \in \mathbb{R}\right\} .
$$

For any ideal $A$ of $\mathcal{O}$, let $\Gamma(A)$ be the principal congruence subgroup of $S L(2, \mathcal{O})$ modulo $A$, given by

$$
\Gamma(A)=\left\{\gamma \in S L(2, \mathcal{O}) \mid \gamma \equiv\left(\begin{array}{ll}
1 & 0 \\
0 & 1
\end{array}\right)(\bmod A)\right\} .
$$

Let $\Gamma_{\infty}(\mathcal{O})$ denote the subgroup of upper triangular matrices of $S L(2, \mathcal{O})$ and $\Gamma_{\infty}(A)=\Gamma_{\infty}(\mathcal{O}) \cap \Gamma(A)$. We shall be primarily concerned with the case $A=\lambda^{3}$. On the congruence subgroup $\Gamma\left(\lambda^{3}\right)$, Kubota [12] showed that

$$
\kappa\left(\left(\begin{array}{ll}
a & b \\
c & d
\end{array}\right)\right)=\left(\frac{c}{d}\right)
$$

is a homomorphism. We will refer to $\kappa$ as the Kubota symbol.

\section{Metaplectic FORms And HeCKe operators}

We will denote by $P$ the following complete set of cusps of $\Gamma\left(\lambda^{3}\right) \backslash \mathcal{H}$ :

$$
P=\left\{\infty, \frac{1}{2}, \frac{1}{i+1}, \frac{1}{i+3}, 1,-1,0,2,-i, \frac{1}{i+2}, i+1, i+3\right\} .
$$

These cusps are in one to one correspondence with double cosets in

$$
\Gamma\left(\lambda^{3}\right) \backslash S L(2, \mathcal{O}) / \Gamma_{\infty}(\mathcal{O})
$$

Here is the corresponding set of representatives, denoted by $\Theta$ :

$$
\begin{aligned}
\Theta & =\left\{\left(\begin{array}{ll}
1 & 1
\end{array}\right),\left(\begin{array}{ll}
1 & 1 \\
2 & 1
\end{array}\right),\left(\begin{array}{cc}
1 & 1 \\
i+1 & 1
\end{array}\right),\left(\begin{array}{cc}
1 & 1 \\
i+3 & 1
\end{array}\right),\left(\begin{array}{ll}
1 & 1 \\
1 & 1
\end{array}\right),\left(\begin{array}{cc}
1 & 1 \\
-1 & 1
\end{array}\right)\right\} \\
& \times\left\{\left(\begin{array}{ll}
{ }_{1}-1
\end{array}\right),\left(\begin{array}{ll}
2 & -1 \\
1
\end{array}\right),\left(\begin{array}{ll}
1 & 1 \\
i & 1
\end{array}\right),\left(\begin{array}{cc}
1 & 1 \\
i+2 & 1
\end{array}\right),\left(\begin{array}{c}
i+1 \\
1
\end{array}\right),\left(\begin{array}{c}
i+3 \\
1
\end{array}\right)\right.
\end{aligned}
$$

Let $z=y^{\frac{1}{2}}\left(\begin{array}{ll}y & x \\ 0 & 1\end{array}\right) \in \mathcal{H}$. Let us define the following theta function:

$$
\theta(z)=y^{\frac{1}{2}} \sum_{\alpha \in \lambda^{-1} \mathcal{O}} \exp \left(\pi i\left\{\operatorname{tr}\left(\chi \alpha^{2}\right)+2 i y|\alpha|^{2}\right\}\right) .
$$


Then we have ([8], Theorem 2.1)

Theorem 2.1. The theta function $\theta(z)$ satisfies the following invariance property:

$$
\theta(\gamma z)=\kappa(\gamma) \theta(z) \quad \forall \gamma \in \Gamma\left(\lambda^{3}\right)
$$

Functions like $\theta(z)$ are called the metaplectic automorphic forms. A metaplectic automorphic form of $\Gamma\left(\lambda^{3}\right)$ is a complex-valued function on $\mathcal{H}$, which is an eigenfunction of certain differential operators, such that $f(\gamma z)=\kappa(\gamma) f(z)$ for all $\gamma \in \Gamma\left(\lambda^{3}\right)$, and certain growth condition at cusps. We will define Fourier coefficients of such a form as follows.

For an element $\rho \in S L(2, \mathcal{O})$, we set

$$
\frac{1}{16} \int_{\mathbb{C} /\left(\lambda^{4}\right)} f\left(\rho\left(\begin{array}{ll}
1 & u \\
0 & 1
\end{array}\right) z\right) e(n u) d u=(\mathbb{N} n)^{-\frac{1}{2}} a_{\rho}(n) W_{f}\left(\left(\begin{array}{ll}
n & \\
& 1
\end{array}\right) z\right),
$$

where $d \mu=\frac{d x d y}{y^{3}}, n \in\left(\lambda^{-4}\right)$ and $W_{f}(z)$ is a certain $G L(2, \mathbb{C})$ Whittaker function which is determined by the eigenvalues of $f$ under certain differential operators. In particular, for $f=\theta$, we have

$$
W_{\theta}(z)=y^{\frac{1}{2}} \exp (-\pi i \operatorname{tr}(x)-2 \lambda y) .
$$

Note that if $\rho^{\prime}=\gamma_{1} \rho \gamma_{2}$ with $\gamma_{1} \in \Gamma\left(\lambda^{3}\right), \gamma_{2}=\left(\begin{array}{cc}u & m \\ 0 & u^{-1}\end{array}\right) \in \Gamma_{\infty}(\mathcal{O})$, then

$$
a_{\rho^{\prime}}(n)=\kappa\left(\gamma_{1}\right) e\left(-m n u^{-1}\right) a_{\rho}\left(u^{-2} n\right) .
$$

This means that the Fourier coefficient defined as above at a cusp $\zeta$ of $\Gamma\left(\lambda^{3}\right) \backslash \mathcal{H}$ depends on the choice of the coset representatives, but in a way clearly understood. Let $\tau_{\rho}(z)$ be the Fourier coefficients of $\theta(z)$. We have ([8], Proposition 2.3):

Theorem 2.2. a) The Fourier coefficients $\tau_{\rho}(n)$ is zero if $n$ is not a square in $K$.

b) Let $p$ be a prime, $p \equiv 1\left(\bmod \lambda^{3}\right)$, and suppose that $(p, n)=1$. Then

$$
\tau_{\rho}\left(n p^{2}\right)=(\mathcal{N} p)^{\frac{1}{2}} \tau_{\rho}(n) .
$$

Now let us turn to the Hecke operators. Let $p$ be a prime of $\mathcal{O},(p, \lambda)=1$. We choose $p$ to be primitive. Let

$$
\zeta_{p}=\left(\begin{array}{ll}
1 & \\
& p^{2}
\end{array}\right)
$$

We decompose the double coset

$$
\Gamma\left(\lambda^{3}\right) \zeta_{p} \Gamma\left(\lambda^{3}\right)=\bigcup \Gamma\left(\lambda^{3}\right) \zeta_{i}
$$

There are only finite number of cosets $\Gamma\left(\lambda^{3}\right) \zeta_{i}$. For each such coset, there are $\gamma_{i}, \delta_{i} \in \Gamma\left(\lambda^{3}\right)$, such that $\zeta_{i}=\gamma_{i} \zeta_{p} \delta_{i}$.

Define the Hecke operator $T_{p^{2}}$, on a metaplectic form $f$ of $\Gamma\left(\lambda^{3}\right)$, as follows.

$$
T_{p^{2}} f=\sum \kappa\left(\gamma_{i}\right) \kappa\left(\delta_{i}\right) f\left(\zeta_{i} z\right)
$$

It may be checked that $T_{p^{2}}$ is well defined and $T_{p^{2}} f$ is still a metaplectic form of $\Gamma\left(\lambda^{3}\right)$. Adopting the convention that $a_{\rho}(n)=0$ if $n \notin\left(\lambda^{-4}\right) \mathcal{O}$, we have the following theorem [2]. 
Theorem 2.3. Let $A_{\rho}(n)$ and $a_{\rho}(n)$ be the Fourier coefficients of $\left(T_{p^{2}} f\right)$ and $f$ respectively, with $f$ a metaplectic form of $\Gamma\left(\lambda^{3}\right)$. Then

$$
A_{\rho}(n)=(\mathbb{N} p) \cdot\left[a_{\rho}\left(n p^{-2}\right)+g(n, p) a_{\rho}(n)(\mathbb{N} p)^{-1}+a_{\rho}\left(n p^{2}\right)\right] .
$$

Proof. The following are the possible representatives $\zeta_{i}$, and the resulting contributions.

i) $\zeta_{i}=\left(\begin{array}{ll}p^{2} & 1\end{array}\right), \kappa\left(\gamma_{i}\right) \kappa\left(\delta_{i}\right)=1$. This term contributes $\mathbb{N} p a_{\rho}\left(n p^{-2}\right)$.

ii) $\zeta_{i}=\left(\begin{array}{c}p b \\ p\end{array}\right), b(\bmod p), \kappa\left(\gamma_{i}\right) \kappa\left(\delta_{i}\right)=\left(\frac{b}{p}\right), b$ is chosen such that $\left(\lambda^{3}\right) \mid b$. This term contributes $g(n, p) a_{\rho}(n)$.

iii) $\zeta_{i}=\left(\begin{array}{cc}1 & \\ p^{2}\end{array}\right), \kappa\left(\gamma_{i}\right) \kappa\left(\delta_{i}\right)=1$. This term contributes $\mathbb{N} p a_{\rho}\left(a p^{2}\right)$.

\section{RANKIN-SELBERG METHOD FOR FORMS OF NONRAPID DECAY}

For each cusp $\zeta \in \Theta$ of $\Gamma\left(\lambda^{3}\right)$, define an Eisenstein series $E_{\zeta}(z, s)$ as

$$
E_{\zeta}(z, s)=\sum_{\gamma \in \zeta \Gamma_{\infty}\left(\lambda^{3}\right) \zeta^{-1} \backslash \Gamma\left(\lambda^{3}\right)} y\left(\zeta^{-1} \gamma z\right)^{2 s} .
$$

Here $z=y^{-\frac{1}{2}}\left(\begin{array}{cc}y & x \\ 0 & 1\end{array}\right), y(z)=y$, and $s$ is a complex number.

$E_{\zeta}(z, s)$ converges absolutely for $\operatorname{Re}(s)$ sufficiently large.

The analytic properties of the Eisenstein series are well understood, and to a large extent are determined by their constant terms.

Let $\vec{E}(z, s)$ stand for the column matrix $\left[E_{\zeta}(z, s)\right]_{\zeta \in \Theta}$. Note that the order of the cusp is important. Let $\zeta_{K}^{\star}(s)=\pi^{-s} \Gamma(s) \zeta_{K}(s)$, where $\zeta_{K}(s)$ denotes the Dedekind Zeta function for the field $K$. It is well known that $\zeta_{K}^{\star}(s)$ has a functional equation, $\zeta_{K}^{\star}(s)=\zeta_{K}^{\star}(1-s)$. Define $e_{\zeta \eta}=e_{\zeta \eta}(y, s)$ to be the constant term of the Fourier expansion of $E_{\zeta}(z, s)$ at cusp $\eta$, that is,

$$
e_{\zeta \eta}=\frac{1}{16} \int_{\mathbb{C} /\left(\lambda^{4}\right)} E_{\zeta}\left(\eta\left(\begin{array}{rr}
x & x \\
1
\end{array}\right) z, s\right) d x .
$$

Then it is easily checked that

$$
e_{\zeta \eta}=\delta_{\zeta \eta} y^{2 s}+\phi_{\zeta \eta}(s) y^{2-2 s}
$$

where

$$
\delta_{\zeta \eta}= \begin{cases}1 & \text { if } \zeta=\eta, \\ 0 & \text { otherwise }\end{cases}
$$

and $\phi_{\zeta \eta}$ are entries of the scattering matrix $\Phi(s)$. The matrix $\Phi(s)$ is given as follows. Let $x=2^{-2 s}, a=\frac{4 x^{3}}{1-x}, b=\frac{2 x^{2}(1-2 x)}{1-x}, c=\frac{x(1-2 x)}{2(1-x)}, d=\frac{1-2 x}{8(1-x)}$. Let

$$
A=\left(\begin{array}{llll}
a & b & c & c \\
b & a & c & c \\
c & c & a & b \\
c & c & b & a
\end{array}\right)_{4 \times 4}, B=\left(\begin{array}{llll}
d & d & d & d \\
d & d & d & d \\
d & d & d & d \\
d & d & d & d
\end{array}\right)_{4 \times 4}
$$


Then,

$$
\Phi(s)=\left(\begin{array}{lll}
A & B & B \\
B & A & B \\
B & B & A
\end{array}\right)_{12 \times 12} \cdot \frac{\zeta_{K}^{\star}(2 s-1)}{\zeta_{K}^{\star}(2 s)}
$$

One has

Theorem 3.1. The vector Eisenstein series $\vec{E}(z, s)$ has a meromorphic continuation to the entire s plane, and satisfies the functional equation

$$
\begin{aligned}
\vec{E}(z, s) & =\Phi(s) \vec{E}(z, 1-s), \\
\Phi(s) \Phi(1-s) & =I .
\end{aligned}
$$

Let

$$
E^{\star}(z, s)=\zeta_{K}^{\star}(2 s) \cdot \sum_{\zeta \in \Theta} E_{\zeta}(z, s)
$$

Then, $E^{\star}(z, s)$ has a constant term, at any cusp $\zeta$,

$$
e(y, s)=\frac{1}{16} \int_{\mathbb{C} /\left(\lambda^{4}\right)} E^{\star}\left(\zeta\left(\begin{array}{cc}
1 & x \\
1
\end{array}\right) z, s\right) d x=\zeta_{K}^{\star}(2 s) y^{2 s}+\zeta_{K}^{\star}(2 s-1) y^{2-2 s},
$$

as $\sum_{\eta \in \Theta} \phi_{\zeta \eta}=\sum_{\eta \in \Theta} \phi_{\eta \zeta}=\frac{\zeta_{K}^{\star}(2 s-1)}{\zeta_{K}^{\star}(2 s)}$. For $E^{\star}(z, s)$, we have the following scalar functional equation.

\section{Corollary 3.1.}

$$
\begin{aligned}
E^{\star}(z, s) & =E^{\star}(z, 1-s), \\
e(y, s) & =e(y, 1-s) .
\end{aligned}
$$

Now let us describe the Rankin-Selberg method for forms not of rapid decay.

Let $F(z)$ be a noncuspidal form on $\mathcal{H}$ invariant under $\Gamma\left(\lambda^{3}\right)$ and for any $\zeta \in \Theta$, let

$$
F(\zeta z)=\Psi_{\zeta}(y)+\mathrm{O}\left(y^{N}\right) \text { as } y \rightarrow \infty, \text { for any } N
$$

where

$$
\Psi_{\zeta}(y)=\sum_{i=1}^{l} \alpha_{i} y^{\beta_{i}} \log ^{n_{i}} y, \quad \alpha_{i}, \beta_{i} \in \mathbb{C}, n_{i} \in \mathbb{Z} .
$$

Let $\mathcal{D}$ be the fundamental domain of $\mathcal{H}$ under $\Gamma\left(\lambda^{3}\right)$. We may choose $\mathcal{D}$ to have the following form:

$$
\mathcal{D}=\mathcal{D}^{\prime} \cup\left\{y^{\frac{1}{2}}\left(\begin{array}{ll}
y & x \\
0 & 1
\end{array}\right) \in \mathcal{H} \mid y>T, x \in \mathbb{C} /\left(\lambda^{3}\right)\right\},
$$

where $\mathcal{D}^{\prime}$ is compact, and $T$ sufficiently large. Define

$$
R(F, s)=\zeta_{K}^{\star}(2 s) \sum_{\zeta \in \Theta} \int_{\Gamma_{\infty}\left(\lambda^{3}\right) \backslash \mathcal{H}}\left[F(\zeta z)-\Psi_{\zeta}(y)\right] y^{2 s} d \mu .
$$


Then we have

Theorem 3.2. $R(F, s)$ has analytic continuation to all $s$ and the functional equation

$$
R(F, s)=R(F, 1-s) .
$$

Proof. Following the basic ideas of [4], 3], we write

$$
R(F, s)=R_{F}(s)+R_{F, \psi}(s)+R_{\psi}(s) .
$$

More precisely,

$$
\begin{aligned}
& R_{F}(s)=\iint_{\mathcal{D}} \sum_{\zeta \in \Theta} F(\zeta z)\left(E^{\star}(\zeta z, s)-e(y, s)\right) d \mu, \\
& R_{F, \psi}(s)=\iint_{\mathcal{D}} \sum_{\zeta \in \Theta}\left(F(\zeta z)-\Psi_{\zeta}(z)\right) e(y, s) d \mu, \\
& R_{\psi}(s)=\zeta_{K}^{\star}(2 s-1) \iint_{\mathcal{D}}\left(\sum_{\zeta \in \Theta} \Psi_{\zeta}(y)\right) y^{2-2 s} d \mu \\
& -\zeta^{\star}(2 s) \iint_{\substack{\gamma \in \Gamma_{\infty}\left(\lambda^{3}\right) \backslash \Gamma\left(\lambda^{3}\right) \\
\gamma \neq I}}\left(\sum_{\zeta \in \Theta} \Psi_{\zeta}(y)\right) y^{2 s} d \mu .
\end{aligned}
$$

Each of $R_{F}(s), R_{F, \psi}(s), R_{\psi}(s)$ has analytic continuation to all $s$ and has functional equation $s \rightarrow 1-s$.

\section{Euler PRoducts}

In this section, we will evaluate the Dirichlet series which appears in the convolutions defined in Section 5. We will prove the convolution has the Euler product, the same as the cusp form [2].

Let $f$ be a metaplectic form on $\mathcal{H}$ of $\Gamma\left(\lambda^{3}\right)$, such that for any $\rho \in S L(2, \mathcal{O})$, we have

$$
\frac{1}{16} \int_{\mathbb{C} /\left(\lambda^{4}\right)} f\left(\rho\left(\begin{array}{ll}
1 & u \\
0 & 1
\end{array}\right) z\right) d \mu=\Psi_{\rho}(y)
$$

where $\Psi_{\rho}(y)$ is of the form

$$
\sum \alpha_{i} y^{\beta_{i}} \log { }^{n_{i}} y, n_{i} \in \mathbb{Z}, \alpha_{i}, \beta_{i} \in \mathbb{C} .
$$

Further, let $f$ be an eigenform of $T_{p^{2}}$, and $\lambda_{p}$ be the eigenvalue, i.e.

$$
T_{p^{2}} f=(\mathbb{N} p) \lambda_{p} f \text {. }
$$

Let $a_{\zeta}(n), \tau_{\zeta}(n)$ be the Fourier coefficients of $f(z), \theta(z)$ at the cusp $\zeta$ respectively. Define

$$
D(s)=\sum_{\zeta \in \Theta} \sum_{\substack{n \in\left(\lambda^{-4}\right) \\ n \neq 0}} \frac{a_{\zeta}(n) \tau_{\zeta}(n)}{(\mathbb{N} n)^{s}} .
$$


We have

\section{Theorem 4.1.}

$$
\zeta_{K, \lambda}(2 s) D(s)=R(s) R_{\lambda}(s),
$$

where $\zeta_{K, \lambda}(2 s)$ is the Dedekind $\zeta$ function of $K$ with the $\lambda$-factor removed, and

$$
\begin{aligned}
R(s) & =\prod_{\substack{p \text { prime } \\
(p, \lambda)=1}} \frac{1}{\left(1-\lambda_{p}(\mathbb{N} p)^{\frac{1}{2}-2 s}+(\mathbb{N} p)^{1-4 s}\right)}, \\
R_{\lambda}(s) & =\sum_{\substack{n \in\left(\lambda^{-4}\right) \\
(n)=\left(\lambda^{i}\right) \text { for some } i}} \frac{a_{\zeta}(n) \tau_{\zeta}(n)}{(\mathbb{N} n)^{s}} .
\end{aligned}
$$

Proof. By (2.8), writing $n=n^{\prime} p^{j}$, with $j \geq 0,\left(n^{\prime}, p\right)=1$, we have

$$
\begin{aligned}
\lambda_{p} a_{\rho}\left(n^{\prime}\right) & =(\mathbb{N} p)^{-\frac{1}{2}}\left(\frac{n^{\prime}}{p}\right) a_{\rho}\left(n^{\prime}\right)+a_{\rho}\left(n^{\prime} p^{2}\right), \\
\lambda_{p} a_{\rho}\left(n^{\prime} p\right) & =a\left(n^{\prime} p^{3}\right), \\
\lambda_{p} a_{\rho}\left(n^{\prime} p^{j}\right) & =a\left(n^{\prime} p^{j-2}\right)+a\left(n^{\prime} p^{j+2}\right) \text { for } j \geq 2 .
\end{aligned}
$$

For prime $p,(p, \lambda)=1$ and for any cusp $\zeta$, using the above relations it is easily checked that

$$
\begin{aligned}
(1- & \left.\lambda_{p} \mathbb{N}^{\frac{1}{2}-2 s}+\mathbb{N} p^{1-4 s}\right) \sum_{\substack{n \in\left(\lambda^{-4}\right) \\
n \neq 0}} \frac{a_{\zeta}(n) \tau_{\zeta}(n)}{(\mathbb{N} n)^{s}} \\
= & \left(1-\lambda_{p} \mathbb{N} p^{\frac{1}{2}-2 s}+\mathbb{N} p^{1-4 s}\right) \sum_{\substack{(n, p)=1 \\
n \in\left(\lambda^{-4}\right) \\
n \neq 0}} \sum_{j=0}^{\infty} \frac{a_{\zeta}\left(n p^{2 j}\right) \tau_{\zeta}\left(n p^{2 j}\right)}{\left(\mathbb{N} n p^{2 j}\right)^{s}} \\
= & \left(1-\mathbb{N}^{-2 s}\right) \sum_{\substack{(n, p)=1 \\
n \in\left(\lambda^{-4}\right) \\
n \neq 0}} \frac{a_{\zeta}(n) \tau_{\zeta}(n)}{(\mathbb{N} n)^{s}} .
\end{aligned}
$$

Removing all the primes, we complete the proof of the theorem.

\section{The gLOBAl Integral AND the FUnCtional EQUATION}

Let $F(z)=f(z) \overline{\theta(z)}$, and $f(z)$ be a noncuspidal form satisfying the conditions in (4.1); then $F(z)$ is a nonmetaplectic form on $\Gamma\left(\lambda^{3}\right)$. Under the assumption that the constant term of $f(z)$ at any cusp $\zeta$ is of the form $\psi_{\zeta}(y)$, as mentioned in Section 4, refer to (4.1), it is easy to see that $F(z)$ satisfies the conditions of Theorem 3.2, because $\theta(z)$ has a constant term (scalar multiple of) $y^{\frac{1}{2}}$, and all Whittaker functions are of rapid decay. For this $F(z)$, define $R(F, s)$ as in (3.7), with $\Psi_{\zeta}(y)=\psi_{\zeta}(y) \cdot\left(\right.$ scalar multiple of $\left.y^{\frac{1}{2}}\right)$. We have

Theorem 5.1. For $\operatorname{Re}(s)$ sufficiently large,

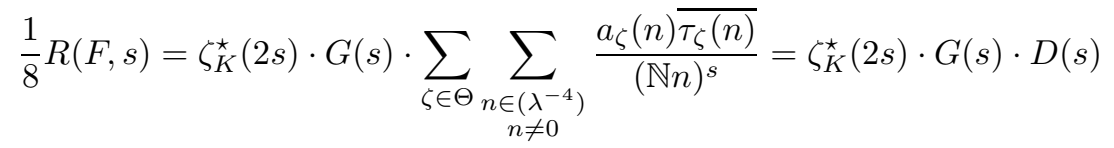


where

$$
G(s)=\int_{0}^{\infty} W_{f}\left(\begin{array}{ll}
y & \\
& 1
\end{array}\right) \overline{W_{\theta}\left(\begin{array}{ll}
y & \\
& 1
\end{array}\right)} y^{2 s-3} d y .
$$

Proof. For each cusp $\zeta$

$$
\begin{aligned}
\iint_{\Gamma_{\infty}\left(\lambda^{3}\right) \backslash \mathcal{H}} & \left(F(\zeta z)-\Psi_{\zeta}(z)\right) d \mu \\
= & \int_{0}^{\infty} \sum_{\substack{n \in\left(\lambda^{-4}\right) \\
n \neq 0}} \frac{a_{\zeta}(n) \overline{\tau_{\zeta}(n)}}{(\mathbb{N} n)} W_{f}\left({ }^{n y}{ }_{1}\right) \overline{W_{\theta}\left({ }^{n y}{ }_{1}\right)} y^{2 s-2} \frac{d y}{y} \\
= & \sum_{\substack{n \in\left(\lambda^{-4}\right) \\
n \neq 0}} \frac{a_{\zeta}(n) \overline{\tau_{\zeta}(n)}}{(\mathbb{N} n)^{s}} \int_{0}^{\infty} W_{f}\left({ }^{y}{ }_{1}\right) \overline{W_{\theta}\left({ }^{y}{ }_{1}\right)} y^{2 s-2} \frac{d y}{y} \\
= & \sum_{\substack{n \in\left(\lambda^{-4}\right) \\
n \neq 0}} \frac{a_{\zeta}(n) \overline{\tau_{\zeta}(n)}}{(\mathbb{N} n)^{s}} \cdot G(s) .
\end{aligned}
$$

Summing over the cusps, we have the desired result.

Now, as proven in $(3.8), R(F, s)$ has the functional equation. Thus $D(s)$ has the functional equation and Euler product.

\section{REFERENCES}

[1] Bump D. and Hoffstein J. Some Euler products associated with cubic metaplectic forms on GL(3), Duke. Math. J. 53 1047-1072 (1986). MR 88d:11044

[2] Bump D. and Hoffstein J. On Shimura's correspondence, Duke. Math. J. 55 661-691 (1987). MR 89c: 11072

[3] Dutta Gupta S. The Rankin-Selberg method on congruence subgroups, Illinois J. Math. (to appear).

[4] Dutta Gupta S. and She X. The GL(2) Rankin-Selberg method for functions not of rapid decay, J. Num. Th. 71 159-165 (1998). MR 99h:11049

[5] Flicker Y. Automorphic forms on covering groups of GL(2), Inv. Math. 57 119-182 (1980). MR 81m:10057

[6] Flicker Y. and Kazhdan D. Metaplectic Correspondence, Publ. Math., IHES 64 53-110 (1986). MR 88d:11049

[7] Friedberg S. Theta series correspondence and modular forms for number fields, In: Modular Forms. Chichester: Horwood, 75-86 (1984). MR 87c:11043

[8] Friedberg S. and Wong S. On the Shimura correspondence for GSp(4), Math. Ann. 290 183-207 (1991). MR 92f:11063

[9] Gelbart S. and Piatetski-Shapiro I. On Shimura's correspondence for modular forms of halfintegral weight, Automorphic forms, Representation Theory and Arithmetic, Bombay, (1979). MR 83b:10032

[10] Goetze T. Euler products associated to metaplectic automorphic forms on the 3-fold cover of GSp(4), Trans. Amer. Math. Soc. 350 No. 3 975-1011 (1998). MR 98e:11061

[11] Hoffstein J. Theta Functions on the n-fold metaplectic cover of GL(2), Invent. Math. 107 61-86 (1992). MR 92k:11049

[12] Kubota T. Ein arithmetischer Satz uber eine Matrizengruppe, J. Reine Angew. Math. 222 55-57 (1966). MR 32:5633

[13] Lieman D. The GL(3) Rankin-Selberg convolution for functions not of rapid decay, Duke Math. J. 69 219-242 (1993). MR 94a:11070 
[14] Niwa S. Modular forms of half integral weight and the integral of certain theta-functions, Nagoya Math. J. 56 147-161 (1974). MR 51:361]

[15] Patterson S. A cubic analogue of the theta series.I, J. Reine Angew. Math. 296 125-161 (1977). MR 58:27795a

[16] Shimura G. On modular forms of half integral weight, Annals of Math. 97 440-481 (1973).

[17] Shintani T. On construction of holomorphic cusp forms of half integral weight, Nagoya Math. J. 58 83-126 (1975). MR 52:10603

[18] Zagier D. The Rankin-Selberg method for automorphic functions which are not of rapid decay, J. Fac. Sci. Univ of Tokyo, Sect. IA. 28 415-437 (1981). MR 83k:10056

Department of Mathematics, Florida International University, Miami, Florida 33199

E-mail address: duttagus@fiu.edu

Financial Data Planning Corporation, 2140 S. Dixie Highway, Miami, Florida 33133

E-mail address: xiaoties@fdpcorp.com 